Revista de Investigación Educativa 26

enero-junio, 2018 | ISSN 1870-5308 | Xalapa, Veracruz

Instituto de Investigaciones en Educación | Universidad Veracruzana

\title{
Uso de Recursos Educativos en Línea en el nivel medio superior: Desarrollo de competencias didácticas del docente
}

\author{
Using Online Educational Resources at the High School level: \\ Development of teaching competences of teachers
}

\author{
Armando Lozano Rodríguez ${ }^{\mathrm{a}}$ \\ José Francisco Zárate Ortiz \\ María Isabel Llaven Aguilar
}

Recibido: 18 de marzo de 2017

Aceptado: 13 de noviembre de 2017

Se presenta un estudio con profesores de nivel medio superior sobre el uso de una herramienta tecnológica llamada Living Class que agrupa recursos educativos en línea. Los objetivos fueron indagar si los profesores que utilizan Living Class desarrollan competencias tecnológicas y de innovación educativa. La metodología fue mixta e imbricada. Se aplicó una encuesta a 130 docentes, además, hubo nueve grupos focales in situ con 27 docentes y se aplicaron 20 entrevistas semi estructuradas por videoconferencia. Los datos obtenidos fueron procesados con el software SPSS para datos cuantitativos y con ATLAs.ti para datos cualitativos. Los hallazgos refieren que tres cuartas partes de los profesores reconocen que la herramienta Living Class ayuda a desarrollar competencias docentes como planificación de tiempo, ajustes en las secuencias didácticas de las clases, desarrollo del modelo de aula invertida, manejo de rúbricas y de aplicaciones tecnológicas novedosas tales como WordDrop, Crosswords, Piktochart, Kahoot, entre otras.

Palabras clave: Innovaciones educativas; recursos didácticos; competencias docentes; ambientes virtuales de aprendizaje.

\footnotetext{
a Doctor, Profesor-Investigador, Instituto Tecnológico de Sonora, México. 凶armando.lozano@itesm.mx

b Doctor, Profesor-Investigador, Instituto Tecnológico y de Estudios Superiores de Monterrey, México. $\bowtie$ jose.zarate@itesm.mx

'Maestra, Coordinadora Académica del Programa para la Inclusión y la Equidad Educativa, Secretaría de Educación del Estado de Chiapas, México.凶 isabella.llaven@gmail.com
} 
A study is presented with professors of upper secondary level on the use of a technological tool called Living Class that groups educational resources online. The objectives were to investigate if the teachers who use Living Class develop technological competences and educational innovation. The methodology was mixed and imbricated. A survey was applied to 130 teachers, in addition, nine focus groups in situ with 27 teachers and 20 semi-structured interviews by videoconference. The data obtained were processed with the spss software for quantitative data and with ATLAS.ti for qualitative data. The findings show that three quarters of teachers recognize that the Living Class tool helps to develop teaching competencies such as time planning, adjustments in the didactic sequences of classes, development of the inverted classroom model, management of rubrics and innovative technological applications such as WordDrop, Crosswords, Piktochart, Kahoot, among others.

Keywords: Technological innovations; didactic resources; teaching competences; virtual learning enviroments.

\title{
Uso de Recursos Educativos en Línea en el nivel medio superior: Desarrollo de competencias didácticas del docente
}

\author{
Using Online Educational Resources at the High School \\ level: Development of teaching competences of teachers
}

\section{Introducción}

$\mathrm{E}$ 1 uso de las Tic (Tecnologías de la Información y la Comunicación) ha tenido un auge considerable en todos los ámbitos de la vida humana, y el de la educación no ha sido ajeno a ello. El empleo de las TIC en educación ha implicado que el docente tenga que implementar métodos de enseñanza diferenciados con base en herramientas atractivas y prácticas que cumplan con la demanda de los estudiantes que están inmersos en estas tecnologías. Por esto mismo, el buen manejo de los recursos educativos u objetos de aprendizaje sustentados en las TIC, puede incidir de una ma- 
nera importante en el aprendizaje. Si se tomara en cuenta la preferencia natural que los alumnos manifiestan hacia la tecnología, se podría sacar mucho provecho para favorecer el proceso de enseñanza-aprendizaje. Sin embargo, si bien hay muchos materiales y recursos educativos multimedia en las páginas web disponibles en Internet, es poco común que los diversos objetos de aprendizaje o materiales didácticos multimedia tengan un sistema que los concentre o que los dirija hacia la enseñanza, o hacia procesos de aprendizaje de manera específica (Chang, 2015).

Los Recursos Educativos en Línea (REL), se refieren a una forma o variante de los llamados Recursos Educativos Abiertos (REA). Los REA es un término propuesto por la Organización de las Naciones Unidas para la Educación, la Ciencia y la Cultura (UnesCO, 2012) que se refiere a Objetos de Aprendizaje (OA) disponibles de manera abierta, gratuita y accesible a través de las TIC y el Internet. Los OA consisten en materiales como videos, documentos (textos), software y otros recursos multimedia para consulta, uso y edición (adaptación) con fines educativos.

Los Rel tienen la ventaja de la diversidad de materiales; son videos, presentaciones y actividades que pueden ser utilizados en diferentes cursos en la escuela y que no necesariamente son OA. Además, el uso de estos recursos educativos permite responder mejor a las necesidades de aprendizaje de estudiantes de nivel medio superior, por la facilidad que provee al maestro para hacerse de materiales que le auxilien en su didáctica; por ejemplo, atender los diferentes estilos de aprendizaje de sus estudiantes y aprovechar también el uso contundente de las TIC que los alumnos de nivel medio superior hacen (Miranda, 2004). Por otro lado, el uso de REL, de acuerdo con Chang (2015), es esencial para el razonamiento individual y para la cognición en los procesos de enseñanza y aprendizaje basados en el uso de la web, ya que permiten el reconocimiento de la información y la adquisición de aprendizajes. Esto quiere decir que con el uso de REL, agrupados en un recurso tecnológico más amplio como es Living Class, se favorecen las relaciones entre los diferentes tipos de representaciones conceptuales y cognitivas y los diferentes estilos de aprendizaje individuales.

Por esto mismo, los procesos de formación escolar contemporáneos han identificado en las TIC y en la virtualización, estrategias claves para fortalecer el aprendizaje de los alumnos. Esto es debido a que los procesos educativos contemporáneos se ven mediados por la producción y uso de contenidos digitales orientados al aprendizaje que implican el diseño, la creación, la usabilidad, la reusabilidad y la accesibilidad de contenidos u objetos de aprendizaje digitales (Herrera, Gelvez \& Sánchez, 2014). Por 
lo mismo, y de acuerdo con Miranda (2004), la introducción y el desarrollo exponencial de las TIC en los ambientes educativos, sumado a la gran generación y uso de contenidos educativos digitales, es decir de REL, han promovido la gran demanda de su uso, tanto en la formación presencial como en ambientes virtuales.

Por ello, las instituciones educativas, presenciales o a distancia, han tenido que ajustar sus métodos de enseñanza para adaptarse a formas educativas más acordes con las características tecnológicas de la sociedad actual, es decir, lograr la formación educativa compatible con las condiciones de uso de las TIC (Salinas, 2004). El uso de las TIC y de los REL en el salón de clases, además de ser una alternativa a la clase tradicional, convierte el aula en un espacio híbrido donde se dan, de manera simultánea, las prácticas formales o tradicionales de enseñanza basados en textos, cuaderno, pizarrón y la exposición del docente, junto con el uso de recursos electrónicos de diversa índole como son las computadoras personales, proyectores, Internet, TIC y los REL (Díaz-Barriga, 2013).

\section{Bases teóricas}

A partir del objeto de estudio antes mencionado, se presentan las siguientes aproximaciones teóricas con respecto a la conceptualización de Recursos Educativos Abiertos, Living Class, y cómo se da su involucramiento en la educación.

\section{1 ¿Qué son los Recursos Educativos Abiertos?}

A partir de las exigencias de la actual Sociedad del Conocimiento ha surgido la necesidad de utilizar diversos recursos en el ámbito educativo. Desde 2001 nace la idea entre los académicos de poder utilizar y compartir de la web para proveer acceso abierto a los materiales educativos existentes en Internet (Bates, 2015). Este desarrollo ha originado infinidad de recursos educativos de todo tipo, que han puesto al alcance de un clic una gran variedad de oportunidades educativas.

El movimiento de los REA dio inicio cuando Johnstone y Poulin (2002), a partir de la iniciativa del Instituto de Tecnología de Massachussets (MIт por sus siglas en inglés), hicieron una primera apreciación global de lo que son todos aquellos recursos de la red que pueden ser recuperados de manera gratuita. Estos autores describen algunos de los motivos de fondo del MIT con esta iniciativa, cómo se han resuelto las 
cuestiones relacionadas con los derechos de propiedad intelectual, y algunos de los desafíos tecnológicos para extender los REA de forma global.

De acuerdo con la Organización para la Cooperación y el Desarrollo Económicos (OCDE, 2007), los REA son un denominador genérico que incluye cursos y programas curriculares, módulos didácticos, guías de estudiante, libros de texto, artículos de investigación, videos, podcasts, herramientas de evaluación, materiales interactivos (como simulaciones), bases de datos, software, aplicaciones (incluyendo las móviles) y cualquier otro material educativo diseñado para su aprovechamiento en la enseñanza y el aprendizaje.

Aun así, hay distintas concepciones de los REA que varían en función del aspecto que se quiere enfatizar, ya sea la reutilización o el ámbito de aplicación (Atkins, Brown \& Hammond, 2007). Por ejemplo, y de acuerdo con Mortera (2010), el término REA, hace referencia a los recursos y materiales educativos gratuitos y disponibles libremente en la World Wide Web (materiales como texto, audio, video, herramientas de software y multimedia, entre otros), y que tienen licencias libres para su producción, distribución y uso en beneficio de la comunidad educativa mundial; particularmente, para su utilización por parte de maestros, padres de familia y alumnos de diversos niveles educativos.

\subsection{Herramienta Living Class}

A partir de la incorporación del uso de las TIC en educación, un grupo de profesores de nivel medio superior del Tecnológico de Monterrey (una universidad multi campus, privada, de México), se propuso seleccionar, recopilar y diseñar actividades que implican el uso ReL disponibles en la web, con el fin de crear experiencias que fomenten el aprendizaje dentro de un ambiente interactivo, retador y colaborativo. Así surgió la iniciativa llamada Living Class en dos cursos de nivel medio superior.

El uso de recursos tecnológicos, tales como los OA y los REL, no es reciente en los profesores de nivel bachillerato de dicha universidad. De hecho, y de acuerdo con lo expresado por los propios profesores participantes de este estudio, luego de varios años utilizando dichos recursos en el salón de clases se ha logrado tener un impacto positivo en el aprendizaje de los alumnos. Por lo que a partir de ello, y en respuesta a procesos de innovación educativa que la institución misma ha promovido entre los docentes, un grupo de profesores propuso compendiar en un solo sitio, embebido en una plataforma electrónica, diferentes actividades educativas de dos materias del pro- 
grama de tercer semestre de nivel medio superior, con la intención de actualizar los materiales y recuperar las mejores actividades y recursos.

La propuesta de una herramienta como Living Class en el nivel superior se sustentó en que esta universidad se plantea, en su modelo educativo, formar a los líderes del Siglo XXI, es decir, a aquellos que enfrentarán retos que aún no existen; es acorde con un modelo educativo que busca transformar la educación tradicional y rescatar la creatividad y curiosidad de los jóvenes para una nueva cultura de aprendizaje (Instituto Tecnológico y de Estudios Superiores de Monterrey [ITESM], 2015). A partir de estas bases surge la idea de Living Class, un proyecto que tuvo como objetivo contribuir en la habilitación de las iniciativas estratégicas del Modelo Educativo Tec 21, aportando valor al proceso de desarrollo de los profesores y la vivencia del alumno.

Living Class es una iniciativa docente del nivel medio superior que consiste en la concentración de Recursos Educativos en Línea (REL) insertados en una plataforma digital educativa (en este caso específico, la plataforma tecnológica Blackboard). De acuerdo con Burgos y Mendoza (2016) Living Class, como herramienta docente para promover un aprendizaje activo, toma un objeto de aprendizaje tipo REL como punto de partida de un tema de estudio correspondiente a una materia o asignatura. Cada objeto de aprendizaje está compuesto por diversos recursos didácticos que para su almacenamiento, clasificación y recuperación se registran en la plataforma tecnológica que la escuela utiliza (Blackboard) haciendo uso de metadatos. El modelo pedagógico de esta iniciativa tiene como referente y guía de orientación el uso de TIC en el salón de clases (ITESM, 2015), así que las competencias, los objetivos y los temas que se encuentran definidos en los planes y programas de estudio oficiales son los ejes medulares para el diseño de cada objeto de aprendizaje con base en REL.

Aunado a lo anterior, Living Class tiene como premisa no sólo recopilar en un mismo lugar diversas actividades y diversos REL para los alumnos, sino que además se fundamenta en las experiencias de aprendizaje que atienden la diversidad de estilos de aprendizaje de los alumnos; y al mismo tiempo, promueve la participación activa de los mismos, reduce la parte tradicional de exposición por parte del profesor y fomenta una clase más interactiva en el aula.

\subsection{Involucramiento en las actividades de aprendizaje}

De acuerdo con Riascos, Ávila y Quintero (2009), respecto al empleo de las TIC, y con ello de los REL en los procesos educativos, hay en general dos perfiles de docentes: 
están aquellos que se resisten a involucrarse en el uso de estos recursos en educación ya sea por renuencia o por cierto temor a la tecnología; y aquellos docentes que están más abiertos a su manejo y apropiación; estos últimos lo hacen por iniciativa propia o por incorporarse a proyectos educativos que involucran a las TIC en educación, y son los que tienen alguna ventaja al mejorar su rol docente y mejorar sus estrategias de enseñanza a través de recursos tecnológicos como las TIC y los REL.

Lo anterior permite visualizar las exigencias que actualmente se dan en la Sociedad del Conocimiento: de implementación de recursos tecnológicos que implican que los docentes cuenten con las competencias necesarias para desempeñar de manera adecuada su rol. Por ello, el docente del siglo XXI requiere usar las TIC para mejorar y transformar las prácticas del aula, y así ayudar a los estudiantes a adquirir y desarrollar los recursos que les permitan desenvolverse eficientemente en el mundo actual; entre ellos, el uso de Internet como fuente de información y de aprendizaje (UNESCO, 2012).

Se debe enfatizar que la información que se obtiene en línea a través de la web no es en sí misma conocimiento, sino que se precisa de la intervención del docente para planear estrategias educativas con la información o con los recursos educativos obtenidos de la red (Díaz-Barriga, 2013). Esto quiere decir que los procesos educativos en el aula, mediados con tecnología y con REL, necesitan de la intervención docente para seleccionarlos, modificarlos y realizar los ajustes necesarios de acuerdo con el programa educativo. De tal modo que es el docente quien da el sentido formativo a los recursos en línea para el aprendizaje de los alumnos, ya que son los docentes quienes tienen las nociones educativas para orientar o conducir el uso de los REL de acuerdo con lo que debe ser aprendido por los alumnos, con las competencias que se busca desarrollar y con las interacciones entre los estudiantes y entre el docente.

En este sentido, los entornos virtuales de aprendizaje (VLE, por sus siglas en inglés), como Living Class, se están volviendo los componentes básicos de la educación contemporánea a distancia, pero también se pueden integrar a un ambiente de aprendizaje físico, como una mezcla de los sistemas presenciales y virtuales (Zulkarnaen, Mat, Khairul \& Mohd, 2016). Por lo tanto, el éxito de la implementación de una herramienta como Living Class depende en gran medida de la aceptación y el uso de un sistema así por parte de los profesores.

El uso de las TiC en educación favorece de varias maneras que los docentes estén a la vanguardia en tecnología educativa. De hecho, es importante que los docentes tengan acceso y dispongan de recursos tecnológicos para su trabajo educativo y de las 
competencias tecnológicas que se requieren para hacer un buen uso de ellas (DíazBarriga, 2013). Por ello, la labor del docente involucra una serie de tareas como el dar seguimiento a las actividades de aprendizaje, dar retroalimentación a los procesos de formación de los alumnos y estar en constante comunicación con ellos, entre otras. En cuanto a las disposiciones y competencias docentes necesarias en la incorporación de las TIC y REL en los procesos educativos, tenemos las siguientes (Riascos et al., 2009):

- Una sólida comprensión de la naturaleza y operación de las Tic.

- Un amplio conocimiento tanto en sistemas tecnológicos como en la solución de problemas relacionados con hardware y software.

- La utilización de herramientas de las TIC y recursos de información para incrementar la productividad, promover la creatividad y facilitar el aprendizaje académico de sus estudiantes.

- El uso de otras herramientas específicas para diferentes contenidos (por ejemplo, software, simuladores, sensores y sondas, calculadoras, graficadoras, ambientes de exploración, herramientas de la web) para apoyar la investigación y el aprendizaje.

- La capacidad de observar y experimentar la utilización de las TIC en sus áreas de estudio especializadas.

- Los docentes que ya han desarrollado estas aptitudes no solo están en capacidad de aplicarlas en sus respectivas instituciones de trabajo, sino que se ven en la obligación de enseñar los conocimientos adquiridos a los nuevos participantes de estos métodos educativos. (p. 139)

Uno de los aspectos más importantes para el uso de los recursos tecnológicos es que sirvan efectivamente en el proceso de enseñanza-aprendizaje en el aula escolar, como ya se dijo. Por un lado, se tiene que, a través de su uso, se favorece que las clases sean más activas e innovadoras; y por otro lado, que se mejore la comunicación entre los alumnos y el docente. Por lo tanto, se puede afirmar que uno de los aspectos que se favorecen con el uso de las Tic y los recursos educativos en línea es que se vuelven un importante apoyo didáctico para el profesor, para transmitir o afianzar conceptos, instruir sobre procedimientos e inculcar valores y actitudes desde estos medios (Gómez, 2008). Sin embargo, se debe tener cuidado de considerar que tanto los docentes como los alumnos pueden hacer un uso superficial, marginado o limitado de los recursos tecnológicos para la educación. 


\section{Planteamiento del problema}

El aprendizaje mediado por tecnología, y específicamente el aprendizaje basado en REL, se ha convertido en el contexto educativo más usual y cada vez más recurrente. Esto es debido al rápido avance y la popularidad de las Tic. Por ello, medir el éxito o describir la percepción de los docentes sobre los sistemas de aprendizaje basados en REL, como Living Class, se ha convertido en un tema importante debido a la proliferación de estos recursos en el contexto educativo. Más aún, aunque el aprendizaje basado en REL ha recibido mucha atención entre los investigadores, pocos estudios se han llevado a cabo para evaluar el éxito y la eficacia, y menos aún para describir la perspectiva de los docentes ante el uso de estos recursos en el aula. Por ello, los métodos de evaluación de la eficacia de estos sistemas de aprendizaje son un tema crítico tanto en la práctica como en la investigación educativa.

Con el fin de abordar el tema, en este estudio se buscaron formas confiables para describir, desde la perspectiva de los docentes, cómo se da el proceso de enseñanzaaprendizaje con una herramienta como Living Class. Este tipo de estudios requiere describir diferentes aspectos en categorías que pueden ser subsumidas dentro de una descripción más amplia para dar una idea de la naturaleza de las interrelaciones entre las categorías y los agentes involucrados (docentes y alumnos), y proponer la ejecución de un mecanismo para evaluar si los resultados y beneficios son acordes con lo esperado en los objetivos (Denzin, 2008).

El problema entonces se concentra en la siguiente pregunta: ¿cómo los profesores que utilizan la herramienta Living Class se vuelven más competentes y más innovadores en el uso de la tecnología?

\section{Metodología}

En este contexto, el método del estudio fue mixto, es decir, de tipo cuantitativo y cualitativo imbricados (Castro \& Godino, 2011; Creswell \& Plano, 2007), cuyos objetivos fueron:

- Indagar si los profesores que emplean Living Class eran más competentes en el uso de la tecnología.

- Indagar si los profesores que emplean Living Class eran más innovadores en su práctica educativa. 
Para los propósitos de esta investigación, el método mixto permitió contrastar, refutar y validar datos cuantitativos y cualitativos que de otra manera no sería posible (Johnson \& Onwuegbuzie, 2004).

\subsection{Participantes}

Los participantes del estudio fueron 130 profesores seleccionados de la base de datos del Tecnológico de Monterrey y que cumplieran con dos criterios: ser profesores de alguna de las tres materias que trabajan con Living Class en el tercer semestre del nivel medio superior y tener al menos dos semestres consecutivos de haber impartido el curso con dicha herramienta. Todos ellos estaban distribuidos en un total de 31 campi a lo largo de la República Mexicana.

\subsection{Instrumentos}

Los instrumentos utilizados para el estudio fueron: 1) Un cuestionario con escala tipo Likert que se aplicó en un lapso de dos semanas a 130 profesores de un total de 186, a través de Internet, utilizando la herramienta Survey Monkey. 2) Nueve sesiones de Focus Group donde participaron 27 profesores de los campi Ciudad de México, Estado de México, Querétaro, Guadalajara y la zona metropolitana de Monterrey (que comprende cinco preparatorias). 3) una entrevista focalizada aplicada a 20 profesores seleccionados de manera propositiva a través de Internet con la herramienta de videoconferencia Webex.

\subsection{Procedimiento}

La totalidad de los profesores fueron invitados a participar en el estudio (grupo focal, entrevista o encuesta), a través de un mensaje de correo electrónico y de la Dirección de su centro de trabajo respectivo. El cuestionario estuvo disponible en línea por un lapso de dos semanas y se le enviaron tres recordatorios al total de la población para exhortarla a participar. En el mismo periodo, se llevaron a cabo los grupos focales in situ en los campi de Querétaro, Guadalajara Ciudad de México y Estado de México del Sistema Tecnológico de Monterrey. Para la zona metropolitana de Monterrey, que agrupa a los profesores de las cinco preparatorias de la localidad, el grupo focal se llevó a cabo a través de videoconferencia con el sistema WebEx. Las entrevistas también se realizaron vía WebEx. 
Los resultados de la encuesta se procesaron con la herramienta Survey Monkey y el paquete estadístico SPss. El índice de confiabilidad de acuerdo con el Alfa de Cronbach fue de 0.927 , lo cual permite asegurar en un nivel aceptable. Los audios de los grupos focales y las entrevistas fueron transcritos para su posterior procesamiento a través del software ATLAs.ti para datos cualitativos. El método de análisis fue la identificación de códigos y categorías para la conformación de familias temáticas que responden directa o indirectamente a los objetivos del estudio. Cada familia representa una categoría de análisis y es complementada con las notas del diario de campo recopiladas durante las visitas a los campi para el grupo focal, y con la inclusión de memos que realizaron los investigadores dentro de la misma herramienta. Los resultados se muestran a continuación.

\section{Resultados}

\subsection{Datos cuantitativos}

La sección de datos sociodemográficos del cuestionario arrojó que $64 \%$ de la población docente considerada para este estudio fue del género femenino y $36 \%$, del masculino. $72 \%$ son profesores y profesoras de cátedra (eventuales), lo cual significa que no tienen un contrato de planta dentro de la institución; $25 \%$ son de planta y $3 \%$ son de media planta o auxiliares planta, es decir, personal administrativo que puede impartir uno o dos cursos como sobrecarga. 93\% de los participantes son adoptadores de Living Class; esto es, son docentes que no participaron en la búsqueda, propuesta y selección de los materiales que conforman la herramienta. Sólo $7 \%$ son diseñadores y, en cierta forma, son los responsables de la configuración actual de Living Class.

En términos de la naturaleza del tipo de programa en el que se llevó a cabo la implementación de Living Class, 70\% de los participantes trabaja en el formato bicultural, $27 \%$ en el formato multicultural y sólo $3 \%$ en el formato internacional. En este sentido, el empleo del idioma inglés se hace indispensable en la mayor parte de los cursos que se utilizan.

Las materias que incluyeron Living Class fueron: La materia y el entorno, con $60 \%$ de la población (que se imparte en español e inglés); Perspectiva del mundo actual (en español e inglés) con 27\%; y Globalization, opportunities and challenges con 14\% (sólo en inglés). $27 \%$ de los profesores participantes cuenta con nivel de licenciatura, $68 \%$ con el grado de maestría y $5 \%$ con grado de doctorado. 
Con base en los propósitos del estudio, se resaltaron seis variables, de un conjunto de dieciocho, sobre las cuales se pretendió indagar la postura de los docentes sobre el uso y participación de Living Class, las cuales se presentan en la Tabla 1.

\section{Tabla 1. Variables de orientación sobre las percepciones de los profesores respecto a Living Class (LC)}

\begin{tabular}{lccc}
\hline \multicolumn{1}{c}{ Variables } & De acuerdo & $\begin{array}{c}\text { En } \\
\text { desacuerdo }\end{array}$ & $\begin{array}{c}\text { Indiferente/ } \\
\text { No sabe }\end{array}$ \\
\hline $\begin{array}{l}\text { Las actividades de LC son motivantes, } \\
\text { novedosas y/o retadoras }\end{array}$ & $64 \%$ & $14 \%$ & $12 \%$ \\
\hline $\begin{array}{l}\text { LC permite implementar el modelo de Aula } \\
\text { Invertida }\end{array}$ & $79 \%$ & $11 \%$ & $10 \%$ \\
\hline LC ayuda al manejo del tiempo en clase & $49 \%$ & $19 \%$ & $32 \%$ \\
\hline LC permite reflexionar sobre la práctica docente & $65 \%$ & $11 \%$ & $24 \%$ \\
\hline LC desarrolla competencias tecnológicas & $59 \%$ & $18 \%$ & $23 \%$ \\
\hline LC aporta recursos y herramientas valiosas & $69 \%$ & $13 \%$ & $18 \%$ \\
\hline
\end{tabular}

Fuente: Elaboración propia.

Las variables con mayor porcentaje de acuerdo por parte de los docentes fueron la de Aula invertida y la de recursos y herramientas valiosas. Sobre esta última, pudiera parecer desconcertante que $13 \%$ de profesores estuviera en desacuerdo. Sin embargo, hay que considerar que un pequeño segmento de la población son profesores con muchos años de experiencia dentro de la institución y que les ha tocado experimentar las distintas fases de adopción de tecnología, por lo que para ellos Living Class no es significativamente de apoyo. Han estado tan acostumbrados al cambio constante que uno más no ha hecho gran diferencia.

Aunado a lo anterior, la variable referente al apoyo que brinda Living Class al profesor sobre el uso eficiente del tiempo en el salón de clase tuvo 19\% de desacuerdo, lo cual puede estar referido al mismo grupo de docentes con experiencia en el uso de distintos tipos de tecnología dentro de la institución. En el mismo sentido, llama la atención que existen porcentajes altos en la columna de indiferencia o en donde los docentes opinaron no saber, sobre todo en las variables de manejo del tiempo en clase y la posibilidad que ofrece la herramienta para reflexionar acerca de la propia práctica 


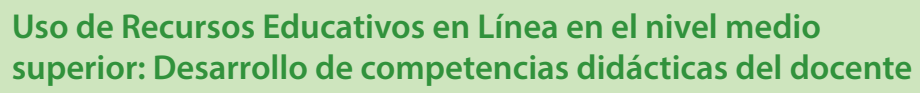

Armando Lozano Rodríguez, José Francisco

Zárate Ortiz, María Isabel Llaven Aguilar

docente. Lo anterior abre nuevas posibilidades de estudio sobre las causas que están originando este fenómeno.

\subsection{Datos cualitativos}

Respecto a los datos cualitativos del estudio y de acuerdo con los objetivos: indagar si los profesores que emplean Living Class son más competentes en el uso de la tecnología y si son más innovadores en su práctica educativa, los datos cualitativos se agruparon en seis categorías, como se puede apreciar en la Tabla 2.

Tabla 2. Categorías de análisis

\begin{tabular}{ll}
\hline Para qué se usa Living Class & Tipo de usos de la herramienta \\
\hline Competencias docentes & Destrezas y saberes que manifiestan los profesores \\
\hline Competencias tecnológicas & Destrezas y saberes sobre el uso de la tecnología \\
\hline Ventajas de Living Class & Características favorables del uso de la herramienta \\
\hline Áreas de oportunidad de Living Class & Errores o dificultades del uso de la herramienta \\
\hline Sugerencias sobre Living Class & $\begin{array}{l}\text { Propuestas de mejora para enriquecer el uso de la } \\
\text { herramienta }\end{array}$ \\
\hline
\end{tabular}

Fuente: Elaboración propia.

Categoría 1: Para qué se usa Living Class, el análisis de los datos indica que, de acuerdo con las percepciones de los docentes, sirve para enriquecer algunas de las funciones docentes y, en consecuencia, su didáctica. Que Living Class es una herramienta muy útil para los docentes porque les ayuda a ampliar y mejorar los recursos didácticos para la clase, lo que facilita el proceso de enseñanza-aprendizaje en alumnos de preparatoria. También, que los temas de las disciplinas pueden ser enseñados de más de una manera, y que la perspectiva no es lineal, sino que puede suponer muchas aristas de aplicación. Todo esto concuerda con lo que Miranda (2004) expone respecto a que el uso de las TIC en el salón de clases es muy acorde con las características del grupo etario, como el de los estudiantes de bachillerato. Lo anterior se articula con que los docentes expresaron que con el uso de Living Class se logra hacer más lúdica la clase, y los alumnos disfrutan más las sesiones dentro del aula cuando hay juego involucrado en sus actividades de aprendizaje. Otro uso relevante de esta 
herramienta es que los docentes que la utilizan consideran que les permite mejorar las secuencias didácticas de sus cursos y con ello, la integración de la información en un solo recurso, en este caso, un recurso tecnológico. Esto es similar a lo que ya fue estipulado por Chang (2015), en el sentido de la eficacia que tiene que los REL estén agrupados para el uso específico con una programación temática (Tabla 3). Esto último es significativo en dos aspectos que también arrojaron los datos: por un lado, que el uso de Living Class ayuda a sincronizar los contenidos del curso con los REL, y por el otro, que se hace un uso mucho más efectivo del tiempo-clase en el aula.

\section{Tabla 3. Usos de Living Class}

\begin{tabular}{ll}
\hline & - Amplía el repertorio didáctico de la clase. \\
Categoría 1: & - Se mejora la planeación didáctica. \\
Para qué se usa Living Class & - Se integra la información y los REL. \\
& - Tiempo efectivo de clases en el aula. \\
& - Clases más lúdicas. \\
\hline
\end{tabular}

Fuente: Elaboración propia.

Categoría 2: Competencias docentes. Los datos obtenidos pueden indicar que el uso de Living Class sí permite el desarrollo de algunas competencias docentes. Entre las principales competencias que los docentes señalaron que les ayuda a desarrollar está la planeación didáctica, al reconocer que cada clase contiene una serie de fases o etapas que pueden reforzarse o intercambiarse. Un aspecto relevante de esto es que al mejorar su planeación didáctica, los docentes incrementan sus formas de contextualizar el contenido del curso (temas) con situaciones más reales que presentan los REL. Otra competencia que los docentes expresan que Living Class les ayuda a desarrollar es que los REL, los documentos de lectura y los demás elementos contenidos en la herramienta, los actualizan, de alguna manera, en su disciplina de estudio (Tabla 4). Otra competencia docente es en relación al uso diversificado de distintas técnicas didácticas que Living Class permite, por ejemplo, el aprendizaje colaborativo (formación de equipos), casos, debates y aula invertida (Flipped Classroom). Pero quizá el área de desarrollo docente más significativo que promueve es que, de acuerdo con los participantes, los profesores cumplen mejor con el compromiso de que sus estu- 
diantes desarrollen en sí mismos habilidades de aprendizaje como la autogestión, la disciplina, la búsqueda y análisis de información y la conexión entre temas. Esto es relevante porque, tal como ya se expuso en el marco teórico, el uso de REL es importante para el razonamiento individual y la cognición en los procesos de aprendizaje basados en el uso de la web, ya que estos permiten el reconocimiento de la información y la adquisición de habilidades para el aprendizaje (Chang, 2015).

\section{Tabla 4. Competencias docentes}

\begin{tabular}{ll}
\hline & - Planeación didáctica. \\
Categoría 2: & Contextualización de los contenidos con ejemplos reales a \\
Competencias docentes & través de REL. \\
& - Actualización docente en su disciplina. \\
& - Uso de diversas técnicas didácticas. \\
& - Desarrollo de habilidades de aprendizaje en los alumnos. \\
\hline
\end{tabular}

Fuente: Elaboración propia.

Categoría 3: Competencias tecnológicas. Un dato interesante que el análisis de los datos arrojó es que Living Class ayuda más a los profesores de nuevo ingreso (con uno y hasta tres semestres consecutivos como docentes) a incorporar recursos tecnológicos en su práctica docente, lo que contrasta un poco con lo que los profesores de mayor antigüedad (más de cinco semestres consecutivos como docentes) expresaron respecto a las competencias tecnológicas. De cualquier manera, en este rubro es posible aseverar que promueve en los docentes algunas competencias para el uso de la tecnología en el aula (Tabla 5). Estas competencias son, por un lado, la exploración de manera intuitiva de nuevas herramientas y aplicaciones contenidas en el diseño de Living Class. Por otro lado, el uso y familiarización de programas (Software) de computadora útiles en la práctica docente, tales como PiktChart, WordDrop, Crossword, Kahoot y Quizzes, entre otros. En el mismo sentido, el uso de Living Class en el aula permite a los docentes usar con más frecuencia aplicaciones (Apps) y sobre todo juegos con fines educativos (ludificación). 
Armando Lozano Rodríguez, José Francisco

Zárate Ortiz, María Isabel Llaven Aguilar

\section{Tabla 5. Competencias tecnológicas}

\begin{tabular}{ll}
\hline & - Incorporación de recursos tecnológicos en la práctica \\
& docente. \\
Categoría 3: & - Exploración intuitiva de nuevas herramientas y aplicaciones. \\
Competencias tecnológicas & $\begin{array}{l}\text { - Uso de programas (Software) educativos. } \\
\text { - Estimulación del aprendizaje con base en juegos de Internet } \\
\\
\text { (Ludificación). }\end{array}$ \\
\hline
\end{tabular}

Fuente: Elaboración propia.

Categoría 4: Ventajas de Living Class (Tabla 6), se presentan las que obtiene el docente cuando usa una herramienta tecnológica como Living Class. Los docentes consideran que les facilita el proceso de enseñanza, les ayuda a administrar mejor el tiempo de la clase y sus propios tiempos de planeación didáctica, lo que repercute en una mejor productividad docente. Además, los docentes aseguran sentirse satisfechos con la labor de enseñar a jóvenes que demandan diversidad didáctica y uso de TIC en el salón de clases. Finalmente, a través de los recursos de Living Class se capta la atención de los alumnos y se les involucra mejor en el proceso de su propio aprendizaje.

\section{Tabla 6. Ventajas educativas de Living Class}

\begin{tabular}{ll}
\hline & - Facilita la enseñanza. \\
Categoría 4: & Mejora la productividad docente. \\
Ventajas de Living Class & - Se enriquece el curso. \\
& - Le capta mejor la atención de los alumnos. \\
& - Es una herramienta sencilla. \\
\hline
\end{tabular}

Fuente: Elaboración propia.

Categoría 5: Áreas de oportunidad de Living Class (Tabla 7) se obtuvieron los resultados que se mencionan a continuación:

Ciertos temas de los programas oficiales de las materias no fueron considerados con recursos en línea por parte de Living Class. Algunos profesores señalaron que esa carencia dificultaba la secuencia y ritmo de la didáctica de las clases. Lo anterior parecía tener un efecto de desconcierto y decepción en los estudiantes. En el mismo 
sentido, varios de los temas tenían un exceso de recursos, mientras que había otros que no tenían ninguno.

Otro de los puntos que fueron señalados como cruciales fue que unos recursos contaban con una traducción deficiente al inglés. Dado que diversas materias son impartidas en ese idioma, parecía que los encargados del diseño de Living Class no habían tenido el cuidado y la pericia de hacer buenas traducciones de los materiales al inglés.

Con respecto a los tutoriales para el uso de la herramienta de Living Class, los profesores reportaron que su ausencia podía ser premeditada para que el mismo docente pudiera navegar a discreción en ella y aprender de manera intuitiva su uso. También reportaron que sería interesante que cada sesión de clase contara con al menos una presentación de Power Point que sirviera como hilo conductor de las actividades y uso de los recursos.

Un detalle que llamó la atención es que algunos profesores sugirieron que para que la herramienta pudiera llamarse Living Class ("clase viva") debería haber actividades interactivas y en tiempo real. De otro modo, sería Dead Class ("clase muerta") porque no existe lo que en teoría debería tener una buena sesión de clase.

Por último, los profesores señalaron la ausencia de autoevaluaciones con resultados inmediatos en Living Class; ese detalle también le imprimiría más vida a la herramienta.

\section{Tabla 7. Áreas de oportunidad de Living Class}

\begin{tabular}{|c|c|}
\hline $\begin{array}{l}\text { Categoría 5: } \\
\text { Áreas de oportunidad de Living } \\
\text { Class }\end{array}$ & $\begin{array}{l}\text { - No todos los temas contenían REL. } \\
\text { - Algunos temas tenían exceso de REL. } \\
\text { - No se cuenta con tutoriales para el uso efectivo de la } \\
\text { - Living Class no cuenta con un sistema de autoevaluación } \\
\text { para los alumnos. }\end{array}$ \\
\hline
\end{tabular}

Fuente: Elaboración propia.

Respecto a esta lista de áreas de oportunidad o faltantes (como indicaron los docentes del estudio) de Living Class, cabe hacer la aclaración que algunas de ellas se refieren a aspectos que están fuera del alcance del diseño de la herramienta; por ejemplo, el que contenga un banco de reactivos y se puedan hacer de manera aleatoria evaluaciones o exámenes. Otras como los materiales en inglés merecerían una evaluación mucho 
más específica, por lo que es difícil establecer si es en sí una desventaja, ya que las percepciones de los docentes participantes son muy particulares. De cualquier manera, las áreas de oportunidad del uso de Living Class como recurso o herramienta tecnológica en el aula deben considerarse para su mejora continua.

Una forma de canalizar las percepciones de los docentes respecto a dichas áreas de oportunidad es atender a las sugerencias que ellos mismos hacen sobre esta herramienta.

Categoría 6: Sugerencias sobre Living Class (Tabla 8). Éstas son: Los docentes involucrados en el uso de Living Class deben participar en un trabajo colegiado y contribuir con ideas, actividades, materiales y diseño de su contenido. Por lo anterior, se recomienda contar con un buzón de sugerencias donde los docentes adoptadores puedan hacer llegar a los diseñadores sus observaciones y comentarios sobre actividades, materiales de apoyo y de contenido.

Los docentes involucrados en el uso de Living Class deben conocer y ser capacitados con suficiente antelación sobre la herramienta, con el fin de que sea efectiva su implementación, lo que incluiría difundir más y mejor su uso para que no quede como un recurso desperdiciado. Respecto al contenido, estructura y diseño de Living Class que los docentes consideran que debe tener un espacio para la creación de un portafolio electrónico de las evidencias del desarrollo de competencias de los alumnos, y que se aplique una evaluación del docente a Living Class.

Brito y Rivero (2014), destacan que las propuestas formativas virtuales, indistintamente del grado de virtualidad en que se ofrezcan, "deben ser evaluadas para asegurar la calidad del proceso de enseñanza-aprendizaje identificando aquellas actividades centrales del proceso que permiten establecer indicadores de calidad significativos, orientar la gestión y el seguimiento del mismo" (p. 21).

\section{Tabla 8. Sugerencias sobre Living Class}

\begin{tabular}{ll}
\hline & - Trabajo colegiado de los docentes para enriquecer el contenido. \\
& - Buzón de sugerencias en LC para comunicación con los \\
& diseñadores. \\
Categoría 6: & Capacitación oportuna. \\
Sugerencias sobre Living Class & Difundir más su uso. \\
& Portafolio de evidencias de las competencias de los alumnos. \\
& - Evaluar LC. \\
\hline
\end{tabular}

Fuente: Elaboración propia. 
Como puede apreciarse, las percepciones de los docentes sobre las desventajas de Living Class y las sugerencias (Categorías 5 y 6) que ellos hacen, giran específicamente en torno a su carácter de herramienta o recurso. En otras palabras, si bien hay aspectos que representan áreas de oportunidad, éstas se deben principalmente a los errores, omisiones y otras fallas en el diseño, construcción y selección de REL específicos de Living Class, y no necesariamente al uso de una herramienta de este tipo como un auxiliar en el salón que ayude a mejorar las clases y promueva el desarrollo de competencias educativas.

\section{Conclusiones}

La herramienta Living Class, en tanto Recurso Educativo en Línea, por su practicidad y disponibilidad ha llevado a muchos docentes a implementarlo en sus aulas de más de una manera, lo cual ha generado opiniones diversas sobre su adopción. Dado que existen varias posibilidades para el abordaje de los temas de contenido, de acuerdo con la percepción de los docentes, se ha sabido sacarles provecho a los recursos de la herramienta a pesar de los inconvenientes derivados de un diseño inacabado y que, en teoría, sigue en perfeccionamiento. No obstante, los resultados del presente estudio han arrojado que los docentes que se han visto más favorecidos con el empleo de Living Class han sido aquellos que poseen poca experiencia o que recién se están incorporando a la actividad de la enseñanza. Los profesores de mayor experiencia no han percibido una mejora significativa en su trabajo docente debido a que han ido evolucionando con el desarrollo de la tecnología y la aparición de recursos que circulan de manera libre y gratuita por Internet. Aunque Living Class agrupa una gran variedad de recursos, a este grupo de profesores con más de diez años de antigüedad no le ha resultado de mucha utilidad.

Uno de los objetivos de este trabajo fue indagar sobre si los docentes desarrollan su creatividad o su competencia de innovación. En palabras de algunos de ellos, Living Class les ha brindado la posibilidad de crear mejores condiciones didácticas con base en la variedad de los recursos incluidos. En otras palabras, al no seguir un programa rígido, se pueden idear o concebir nuevas maneras de explorar o abordar un contenido, haciendo uso no solamente de un recursos específico (un video o una aplicación electrónica), sino también de una técnica didáctica en particular, por ejemplo, la del debate en materias como Química o Biología, que por la misma naturaleza de la disciplina, difícilmente se requieren. 
El otro objetivo fue indagar si los profesores desarrollan competencias docentes derivadas del uso de Living Class. Al respecto, los resultados señalan que un gran número de se pudo familiarizar con la propuesta de las rúbricas como medio para entender mejor el proceso de evaluación, dado que no lo tenían considerado. También, el empleo de técnicas didácticas nunca antes vistas o aplicadas en los contextos de sus disciplinas, y por último, la oportunidad de conocer nuevas y mejores herramientas electrónicas para organizar, procesar o presentar información (PiktChart, WordDrop, Crossword y Quizzes).

Para dar respuesta a la pregunta de investigación, se puede mencionar que uno de los aspectos más significativos del presente estudio es que los profesores ven con mucho aprecio los esfuerzos institucionales por brindar las mejores oportunidades de enseñanza en términos de apoyos tecnológicos. La competencia tecnológica se vio incrementada solamente en los profesores novatos y no así en los de mayor experiencia en la institución. Lo anterior se debe a que en el pasado siempre ha existido en la institución el uso de herramientas tecnológicas diversas, sin un ordenamiento particular como lo presenta ahora Living Class. Asimismo, el tema de la innovación está presente en el uso cotidiano de nuevas herramientas que día a día se van incorporando a Living Class. Sin embargo, la estrategia institucional no ha resultado ser la mejor para llevar a cabo la implementación del proyecto. En palabras de Zabalza-Beraza y ZabalzaCerdeiriña (2012), los proyectos de innovación educativa deben descender por etapas secuenciadas desde el grupo que concibe el proyecto hasta la base de la pirámide. Para asegurar lo anterior, se requiere un proceso puntual de seguimiento y evaluación.

\section{Lista de referencias}

Atkins, D. E., Brown, J., \& Hammond, A. L. (2007). A Review of the Open Educational Resources (OER) Movement: Achievements, Challenges, and New Opportunities (Reporte). Recuperado de https://www.hewlett.org/wp-content/ uploads/2016/08/ReviewoftheOERMovement.pdf

Bates, T. (2015). Teaching in a digital age. Canadá: вC Campus.

Brito, J. G., \& Rivero, M. E. (2014). El proceso de producción en la construcción de ambientes virtuales de enseñanza, aprendizaje y comunicación: indicadores para evaluar su calidad. Virtualidad, Educación y Ciencia, 5(8), 18-28.

Burgos, J. V., \& Mendoza, E. E. (2016). Living Class: Modularización del aprendi- 
zaje en la planeación de clase. Memorias del Congreso Internacional de Innovación Educativa, 3(3), 2256-2266. Recuperado de https://drive.google.com/ file/d/1JqxiFlk2-nR3r8bcTB6_HwTo_VtWWnRk/view

Castro, W. F., \& Godino, J. D. (2011). Métodos mixtos de investigación en las contribuciones a los simposios de la SEIEM (1997-2010). En M. Marín, G. Fernández, L. Blanco \& M. M. Palarea (Eds.), Investigación en Educación Matemática XV (pp. 99-118). Ciudad Real: Sociedad Española de Investigación Matemática.

Chang, H.-J. (2015). Beyond the Representation: Cognition in Manipulative Learning Objects within Learning Simple Equations. International Journal of Information and Education Technology, 5, 855-859.

Creswell, J., \& Plano, V. (2007). Designing and conducting mixed methods research. California: SAGE.

Denzin, N. K. (agosto, 2008). Los nuevos diálogos sobre paradigmas y la investigación cualitativa. Un compromiso en la relación universidad-sociedad. Reencuentro, $52,63-76$

Díaz-Barriga, Á. (junio-septiembre, 2013). Tic en el trabajo del aula. Impacto en la planeación didáctica. Revista Iberoamericana de Educación Superior, 4(10). Recuperado de http://148.215.2.11/articulo.oa?id=299128588003

Gómez, G. (2008). El uso de la tecnología de la información y la comunicación y el diseño curricular. Educación, 32, 77-97. Recuperado de http://www.redalyc. org/articulo.oa?id $=44032107$

Herrera, J. F., Gelvez, N. Y., \& Sánchez, J. M. (2014). Iniciativas de estandarización en la Producción de Objetos virtuales de aprendizaje. Journal of Information Systems and Technology Management, 11(3), 677-715. Recuperado de http://www.jistem.fea.usp.br/index.php/jistem/article/view/10.4301\%252 FS1807-17752014000300009

Instituto Tecnológico y de Estudios Superiores de Monterrey. (2015). Plan Estratégico 2020. Recuperado de http://sitios.itesm.mx/webtools/planestrategic02020/

Johnson, R., \& Onwuegbuzie, A. (2004). Mixed Methods Research: A research paradigm whose time has come. Educational Research, 33(7), 14-26.

Johnstone, S., \& Poulin, R. (2002). Technology: What Is OpenCourseWare and Why Does It Matter? Change, 34(4), 48-50. Recuperado de http://www.jstor.org/ stable/40177912

Miranda, A. (2004). De los ambientes virtuales de aprendizaje a las comunidades de aprendizaje en línea. Revista Digital Universitaria, 5(10), 2-14. 
Mortera, F.J. (2010). Implementación de Recursos Educativos Abiertos (REA) a través del portal temoa (Knowledge Hub) del Tecnológico de Monterrey, México. Formación Universitaria, 3(5), 9-20. doi:10.4067/So718-50062010000500003

Organización de las Naciones Unidas para la Educación, la Ciencia y la Cultura. (Junio, 2012). 2012 Paris Oer Declaration. 2012 World Open Educational Resources (OER) Congress. París. Recuperado de http://www.unesco.org/new/fileadmin/MULTIMEDIA/HQ/CI/CI/pdf/Events/Paris\%2oOER\%20Declaration_01.pdf

Organización para la Cooperación y el Desarrollo Económicos. (2007). Giving Knowledge for Free: The Emergence of Open Educational Resources. Recuperado de http://www.oecd.org/edu/ceri/38654317.pdf

Riascos, S. C., Ávila, G. P., \& Quintero, D. M. (2009). Las TiC en el aula: percepciones de los profesores universitarios. Educación y Educadores, 12, 133-157. Recuperado de http://educacionyeducadores.unisabana.edu.co/index.php/eye/article/view/1536

Salinas, J. (diciembre, 2004). Innovación docente y uso de las TIC en la enseñanza universitaria. Revista de Universidad y Sociedad del Conocimiento, RUSC, 1(1). Recuperado de http://www.uoc.edu/rusc/dt/esp/salinas1104.pdf

Zabalza-Beraza, M. A., \& Zabalza-Cerdeiriña, M. A. (2012). Innovación y cambio en las instituciones educativas. Barcelona, España: Homo Sapiens.

Zulkarnaen, K., Mat, D., Khairul, A., \& Mohd, H. (junio, 2016). The Relationship among Student's Domain of Learning Development Implementing Virtual Learning in Higher Learning Institutions. International Journal of Information and Education Technology, 6(6), 418-421. Recuperado de http://www.ijiet. org/vol6/725-Too19.pdf 